\title{
Transportation Network Design considering Morning and Evening Peak-Hour Demands
}

\author{
Hua Wang, ${ }^{1}$ Gui-Yuan Xiao, ${ }^{2}$ Li-Ye Zhang, ${ }^{3}$ and Yangbeibei $\mathrm{Ji}^{4}$ \\ ${ }^{1}$ School of Economics and Management, Tongji University, Shanghai 200096, China \\ ${ }^{2}$ College of Civil and Architectural Engineering, Guilin University of Technology, Guilin 541004, China \\ ${ }^{3}$ School of Traffic and Transportation Engineering, Changsha University of Science and Technology, Changsha 410076, China \\ ${ }^{4}$ School of Management, Shanghai University, Shanghai 200444, China
}

Correspondence should be addressed to Li-Ye Zhang; liyezhang.uf@gmail.com

Received 18 December 2013; Accepted 17 January 2014; Published 10 March 2014

Academic Editor: X. Zhang

Copyright (C) 2014 Hua Wang et al. This is an open access article distributed under the Creative Commons Attribution License, which permits unrestricted use, distribution, and reproduction in any medium, provided the original work is properly cited.

\begin{abstract}
Previous studies of transportation network design problem (NDP) always consider one peak-hour origin-destination (O-D) demand distribution. However, the NDP based on one peak-hour O-D demand matrix might be unable to model the real traffic patterns due to diverse traffic characteristics in the morning and evening peaks and impacts of network structure and link sensitivity. This paper proposes an NDP model simultaneously considering both morning and evening peak-hour demands. The NDP problem is formulated as a bilevel programming model, where the upper level is to minimize the weighted sum of total travel time for network users travelling in both morning and evening commute peaks, and the lower level is to characterize user equilibrium choice behaviors of the travelers in two peaks. The proposed NDP model is transformed into an equivalent mixed integer linear programming (MILP), which can be efficiently solved by optimization solvers. Numerical examples are finally performed to demonstrate the effectiveness of the developed model. It is shown that the proposed NDP model has more promising design effect of improving network efficiency than the traditional NDP model considering one peak-hour demand and avoids the misleading selection of improved links.
\end{abstract}

\section{Introduction}

The transportation network design problem (NDP) is characterized as one of the most important and challenging optimization problems in the transportation system [1]. It aims to improve the network system efficiency by expanding link capacities on existing roads or building new roads/lanes for the network. In general, the NDP problem can be well formulated by a bilevel programming model, in which the Stackelberg behavior between network planner and network users is characterized. The upper level problem is to optimize a set of design objectives (e.g., minimizing total travel time) for the urban transportation system by setting link capacity expansion scheme with some necessary constraints (e.g., financial budget constraint). The lower level problem is to describe the user's behavior in terms of path/mode choice, departure time choice, and origin/destination choice.
After a pioneering work of Abdulaal and Leblanc [2], more and more researchers have paid attention to the NDP problem with focus on advanced model formulation and effective algorithm design. The achievements made before last century on the NDP studies can be found in two comprehensive reviews: Magnanti and Wong [3] and Yang and Bell [4]. In recent decade, we have also witnessed a large number of emerging advances on the NDP studies. These new advances are pertinent to uncertain parameters (e.g., stochastic demand and capacity variation), sustainability constraints, and distinct time dimension. Uncertainty is one underlying and important feature of travel activity and plays a critical role in network design and planning policy. To avoid unnecessary risks and possibly misleading outcomes, demand uncertainty and/or capacity variation have been taken into account in the NDP problem (e.g., $[5,6])$, by introducing expected value model and robust optimization model. Two typical 
approaches are used to model the stochastic traffic flows under uncertain traffic conditions. One is to develop a two-stage formulation by means of scenario-construction approach, where a number of finite scenarios of uncertain parameters with known probability distribution will be generated (e.g., [7, 8]). Another way, called probability-analytical method, is to derive a reliability-based traffic assignment, where the users follow probabilistic user equilibrium under uncertain traffic conditions (e.g., [9-11]).

Time dimension, as another important factor, has also attracted the researchers' attention in recent NDP studies. Three scales of time dimension can be considered in the NDP problem: short-term/real-time (seconds), mediumterm (days), and long-term (years). The NDP considering time dimension is defined as time-dependent NDP, which can be specifically classified into multiperiod NDP and dynamic NDP according to different scales of time dimension. The former is generally to analyze the NDP problem taking into account a long-term spanning construction and maintenance of transport infrastructure [12-15]. The latter is to precisely characterize the real-time traffic dynamics and unsteady-state conditions in the NDP by introducing dynamic traffic assignment. Hopefully it can be used to estimate microinteraction among adjacent links and thereby identify and examine the possible bottleneck $[6,16,17]$. A comprehensive review of recent developments on the NDP studies can be referred to Meng et al. [18].

By retrieving and reviewing the NDP literature, especially the valuable reviews across several decades $[3,4,18,19]$, it can be found that almost all NDP studies assume that an NDP model based on one peak-hour O-D demand distribution is capable and effective to explain the real traffic patterns. For urban traffic, there always are two peaks of a daily traffic flow corresponding to morning commute and even commute, respectively, on weekday [20]. For commuting travel trips, the repeatability of hourly fluctuation of traffic flow gives rise to a comparative stable peak-hour demand. But such repeatability of hourly variation of traffic flow does not mean the same O$\mathrm{D}$ demand distributions in the morning and evening peaks.

Although the daily commuters have high repetitive travel activities, the peak-hour O-D demand distribution on the network will be different in the morning and evening peaks. This fact has been recognized and emphasized in the transportation network modeling problem, such as commuting pattern analysis [21] and road tolling and parking fee optimization $[22,23]$. It is always assumed that the O-D demand matrix is asymmetric diagonal for the morning and even peaks, namely, $q_{o d}=q_{d o}$, if only daily regular commuters are considered. The O-D demand matrix used in the network planning and design comes up with the peak-hour traffic survey data (e.g., link traffic volumes) in either morning or evening peak. In practice, the traffic volumes in the morning peak may largely differ from that of evening peak commute, which has been demonstrated by some empirical studies (e.g. $[20,24])$. The O-D demand matrices thus may also be different for two peaks because they are estimated based on the corresponding peak-hour traffic volumes. In other words, the travelers in two commuting peaks would make different travel decision in terms of both path choice and departure

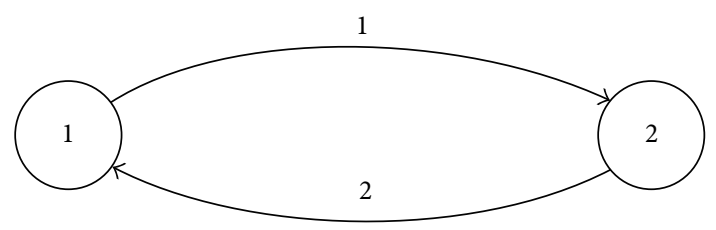

Figure 1: An illustrative example.

time choice [25]. Therefore, the NDP considering one peakhour O-D demand might be misleading and even incorrect. On the one hand, the O-D demand distribution on a real network is asymmetric due to different traffic patterns in the morning and evening peaks, though the morning and evening round commuting trips can be assumed to be equal. We should overlook demand uncertainty and diverse spatialtemporal characteristic of travel activities in two peaks. For example, the noncommuting trips in morning peak (e.g., shopping, going to hospital, and tourism) may not return back to the origin places in evening peak. Similarly, part of activities for the travelers who travel in evening peak do not commence in the morning and evening peaks but other traveling periods. Therefore, which peak-hour commute pattern should be used to estimate the O-D demand matrix is a controversial and intractable issue.

On the other hand, network structure and link sensitivity could have important impact on the network performance evaluation for two peaks. Even though the O-D pairs and their demands can be assumed to be symmetric in two peaks, namely, $q_{o d}=q_{d o}$, the path choices in two commute peaks may be still different, which largely depends on the network topological structure. If different paths/links are chosen in two peaks, network situation in terms of traffic congestion would show large difference between two peaks. In this respect, the NDP considering one peak-hour O-D demand may generate misleading and even wrong outcomes. More importantly, link sensitivity of the NDP effect should not be underestimated and overlooked under different peaks. Take a simple network comprising of two links shown in Figure 1 as an example. Assume that $q_{o d}$ in the morning peak is equal to $q_{d o}$ in the evening peak. Consider two O-D pairs, $q_{12}=30, q_{21}=10$ in the morning peak and $q_{12}=10$, $q_{21}=30$ in the evening peak. Links 1 and 2 have equal capacities of $20 \mathrm{veh} / \mathrm{h}$ and free flow travel time of 1 minute. The available budget invested on link capacity expansion is set as 20 equivalently. BPR function is used to measure the link travel time performance.

It is not difficult to see that link 1 will be improved by adding 20 units in the NDP considering morning peak-hour demand, and link 2 will be improved by expanding 20 units in the NDP considering evening peak-hour demand. The total travel times on the network for these two NDPs are equal, namely, 104.305 minutes. In the NDP simultaneously considering morning and evening peak-hour demands, both links 1 and 2 will be improved by adding 10 units, respectively, and thereby the total travel time on the network will be reduced to 89.037 minutes. Evidently, the NDP considering one peak-hour demand matrix is not the promising scheme 
to improve transportation system performance. Therefore, it is necessary to develop a new network design model in order to avoid misleading decision-making.

To address the above problems, we develop a network design model simultaneously taking into account morning and evening peak-hour demands. The traditional NDP model considering one peak-hour O-D demand has been regarded as a special case of the proposed model. The proposed model is formulated as a bilevel programming model with objective to minimize the weighted sum of total travel times of the traffic patterns in the morning and evening peaks. In the lower level, network users are assumed to follow the user equilibrium principle of their route choice decision. The proposed model is finally transformed into an equivalent mixed integer linear programming (MILP) so that a global optimum can be obtained by means of MIP solvers (e.g., CPLEX).

The rest of the paper is organized as follows. In the next section, the new NDP model is formulated by simultaneously considering the morning and evening peal-hour O-D demand distributions. Then, an equivalent MILP model is introduced. Numerical examples are discussed in Section 4. Finally, conclusions and further studies are given in Section 5.

\section{The Model}

This section builds a network design model simultaneously considering morning and evening peak-hour demand matrices. Consider a directed transportation network, $G(N, A)$, comprising of a set $N$ of nodes and a set $A$ of directed links. Since traffic flow patterns in two peaks will be involved, the following notations are defined for two peaks, respectively, which are summarized in the Appendix (Notations).

The link travel time in the morning or evening peak is assumed to be continuous, convex, and strictly increasing function of its own link flow, such as the widely-applied BRP function. Meanwhile, our focus is put on the deterministic NDP problem with fixed demand.

2.1. Model Formulation. Similar to the traditional NDP model taking into account one peak-hour demand matrix, a bilevel program is formulated to model the proposed NDP problem considering two peak-hour demand matrices in that the Stackelberg behavior between network planner and network users can be well characterized. In the upper level, the network design objective is to minimize the weighted sum of total travel times (TTC) of the users traveling in the morning and evening peaks. The lower level is the user equilibrium traffic assignment problem, which is used to characterize the user's route choice behavior. The NDP formulation is given below.

\section{Upper Level}

$$
\min _{\mathbf{f}^{m}, \mathbf{f}^{e}, \mathbf{x}} \mathrm{TTC}=\alpha \sum_{w \in W^{m}} \mu_{w}^{m}\left(\mathbf{f}^{m}, \mathbf{x}\right) q_{w}^{m}+\beta \sum_{w \in W^{e}} \mu_{w}^{e}\left(\mathbf{f}^{e}, \mathbf{x}\right) q_{w}^{e},
$$

subject to

$$
\begin{aligned}
& x_{a} \geq 0, \quad a \in \bar{A}, \\
& \sum_{a \in \bar{A}} b_{a}(\mathbf{x}) \leq B .
\end{aligned}
$$

Constraint (2) means a nonnegative link capacity expansion and (3) is the total invested budget constraint. The construction cost for each improved link, $b_{a}\left(x_{a}\right)$, can be approximately estimated by a linear function:

$$
b_{a}\left(x_{a}\right)=\gamma_{a} x_{a}, \quad a \in \bar{A} .
$$

The traffic flow patterns, $f^{m}(\mathbf{x}), f^{e}(\mathbf{x}), \mu_{w}^{m}(\mathbf{x})$, and $\mu_{w}^{e}(\mathbf{x})$ can be obtained by solving the lower level traffic assignment problems in the morning and evening peaks.

\section{Lower Level}

(1) User equilibrium model in the morning peak is as below:

$$
\min _{\mathbf{x}} \sum_{a \in A} \int_{0}^{v_{a}^{m}} t_{a}^{m}(\mathbf{x}, \omega) d \omega
$$

subject to

$$
\begin{gathered}
v_{a}^{m}=\sum_{w \in W^{m}} \sum_{r \in R_{w}^{m}} f_{r, w}^{m} \delta_{a r, w}^{m}, \quad a \in A, \\
\sum_{r \in R_{w}^{m}} f_{r, w}^{m}=q_{w}^{m}, \quad w \in W^{m}, \\
f_{r, w}^{m} \geq 0, \quad r \in R_{w}^{m}, w \in W^{m} .
\end{gathered}
$$

(2) User equilibrium model in the evening peak is as below:

$$
\min _{\mathbf{x}} \sum_{a \in A} \int_{0}^{v_{a}^{e}} t_{a}^{e}(\mathbf{x}, \omega) d \omega,
$$

subject to

$$
\begin{gathered}
v_{a}^{e}=\sum_{w \in W^{e}} \sum_{r \in R_{w}^{e}} f_{r, w}^{e} \delta_{a r, w}^{e}, \quad a \in A, \\
\sum_{r \in R_{w}^{e}} f_{r, w}^{e}=q_{w}^{e}, \quad w \in W^{e}, \\
f_{r, w}^{e} \geq 0, \quad r \in R_{w}^{e}, \quad w \in W^{e} .
\end{gathered}
$$

It should be noted that all travelers in the morning and evening peaks will complete their travel journeys on the same transportation network; that is, the link capacity expansion scheme would be designed and served for the travelers in both morning and evening peaks. Hopefully, it is intuitive that the travelers in both morning and evening peaks will all benefit from the NDP scheme.

We can easily see that, if $\alpha=1, \beta=0$, the proposed model is equivalent to the traditional NDP model considering morning peak-hour demand distribution in that the evening peak-hour traffic patterns are not considered in the objective function. Similarly, if $\alpha=0, \beta=1$, the proposed model is equivalent to the traditional NDP model considering evening peak-hour demand distribution, since the morning commute pattern has no impact on the objective function. 
2.2. Extensions of considering Elastic Demand and Uncertain Demand. The proposed model can be also extended to account for the elastic demand and/or uncertain demand. We here give a brief discussion of these extensions. In reality, whether a potential traveler decides to finish her/his travel journey in great extent depends on the traffic congestion on the network, no matter what commute peak is considered. The O-D demand thus can be assumed to be a function of the $\mathrm{O}-\mathrm{D}$ travel cost. Take the O-D travel demand in the morning peak as an example:

$$
q_{w}^{m}=d_{w}\left(\mu_{w}^{m}\right), \quad w \in W^{m},
$$

where $d_{w}(\cdot)$ is the demand function between O-D pair $w \in$ $W^{m}$ and its inverse function is represented by $d_{w}^{-1}(\cdot)$. In general, $d_{w}(\cdot)$ is assumed to be a positive, continuously differentiable, and strictly decreasing function with respect to shortest path cost $\mu_{w}^{m}$, such as negative exponential function.

It is not difficult to deal with the proposed NDP model with elastic demand since the traffic assignments for two peaks with elastic demand can be easily solved by a supernetwork method or an improved Frank-Wolfe algorithm in Sheffi [26].

Another interesting extension is to take into account of the uncertain travel demands in two peaks. The importance of considering uncertainties in the NDP is to avoid the unnecessary risks and misleading policies. The O-D demand in either morning commute peak or evening commute peak will fluctuate in future. To capture these impacts, we can develop a general stochastic NDP according to the below framework:

$$
\min _{\mathbf{V}, \mathbf{x}} F(\mathbf{V}, \mathbf{x}, \boldsymbol{\Xi})
$$

subject to

$$
\begin{gathered}
\mathbf{V} \in \Omega(\mathbf{x}, \boldsymbol{\Xi}) \\
(\mathbf{V}, \mathbf{x}) \in \Theta .
\end{gathered}
$$

The bold notations, $\mathbf{V}, \boldsymbol{\Xi}$, are random variables, which denote stochastic link flows and uncertain demands in the morning and evening peaks. Notation $\Theta$ denotes the set of additional constraints of variables $\mathbf{V}$ and $\mathbf{x}$, and $\Omega(x, \boldsymbol{\Xi})$ is the set of feasible link flows on stochastic network.

Two modeling methods can be used to characterize the morning and evening peak-hour demand uncertainties in the NDP problem. One is to develop the probabilistic or reliability-based user equilibrium model of deriving the stochastic traffic flow patterns in the morning and evening peaks and then embed them into the upper level optimization of the NDP problem. For such probabilistic user equilibrium model, it can be referred to Lo et al. [9], Shao et al. [10], and Wang et al. [11]. Another way is to formulate a twostage NDP model, where the uncertainties of two peakhour demands can be captured by the random samples generated by some scenario-construction methods, such as sample average approximation $[7,8]$. The main difference between two modeling approaches and the detailed formulation framework can be found in Meng et al. [18].

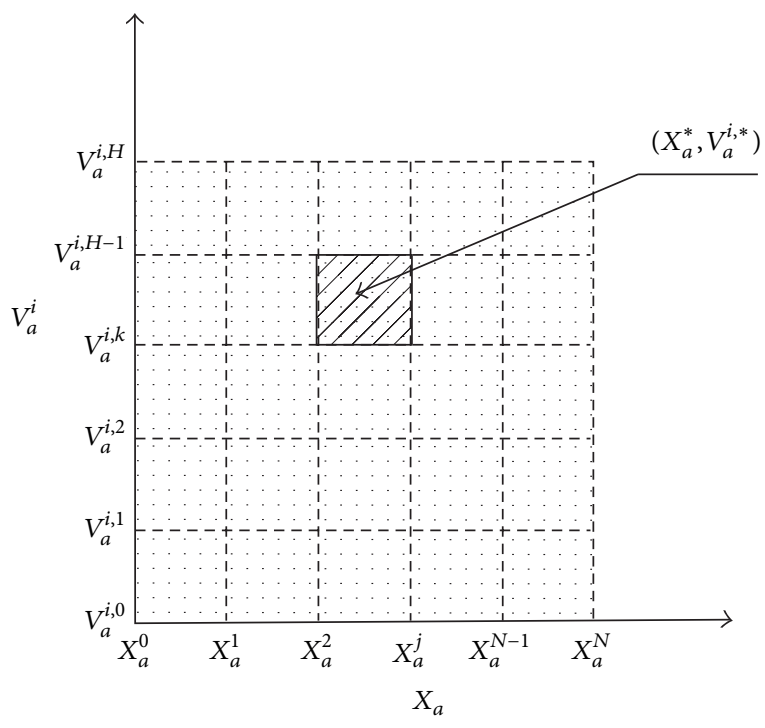

FIGURE 2: Discretize binary space into feasible regions.

\section{The Equivalent MILP Model}

This study focuses on the NDP problem with two peakhour demand impacts and on examining the design effects between the proposed model and the conventional one with one peak-hour demand matrix. In order to precisely compare the network design effects, it would be better to solve the NDP model by a global solution algorithm. We here use a global solution algorithm proposed by Wang and Lo [27] by transforming the bilevel NDP model into single-level MILP. The transformation of MILP includes linearization of design objective function, link travel time function, and other side constraints.

For the sake of simplicity, the fixed demand is considered and the BRP function is used to measure the congestion effect of the link travel time, namely,

$$
t_{a}^{i}\left(v_{a}^{i}, x_{a}\right)=t_{a}^{0}\left(1+0.15\left(\frac{v_{a}^{i}}{c_{a}^{0}+x_{a}}\right)^{4}\right), \quad i=\{m, e\}
$$

where $t_{a}^{i}$ and $v_{a}^{i}$ are used to represent the link travel time and link flow for the morning peak if $i=m$ or for the evening peak if $i=e$. Hereafter, we briefly revisit the transformation of MILP.

3.1. Linearization of Link Travel Time Function. It can be seen in (12) that the link travel time function for each commute peak is a function of link flow in the peak and design variable, namely, link capacity expansion. Similar to Wang and Lo [27], Luathep et al. [28], and Zhang and Van [29], the binary space in terms of link flow and link capacity expansion can be divided into $H \times N$ feasible regions as shown in Figure 2 . Let $v_{a}^{i, l}, v_{a}^{i, u}$ be the lower and upper bounds of $v_{a}^{i}$, and let $x_{a}^{l}$, $x_{a}^{u}$ be the lower and upper bounds of $x_{a}$. For all discretized intervals, we have $v_{a}^{i, l}<K_{a, h}^{i}<K_{a, h+1}^{i}<v_{a}^{i, u}$ and $x_{a}^{l}<$ $L_{a, n}<L_{a, n+1}<x_{a}^{u}, h \in\{1,2, \ldots, H\}, n \in\{1,2, \ldots, N\}$, and $i=\{m, e\}$. 
For any feasible region $[h, n]$, the link travel time function (12) can be approximated as a linear function by Taylor expansion:

$$
\begin{array}{r}
t_{a}^{i}\left(v_{a}^{i}, x_{a}\right)=a_{a}^{i, h, n} v_{a}^{i}+b_{a}^{i, h, n} x_{a}+c_{a}^{i, h, n}, \\
\text { if } K_{a, h}^{i} \leq v_{a}^{i} \leq K_{a, h+1}^{i}, L_{a, n} \leq x_{a} \leq L_{a, n+1},
\end{array}
$$

where coefficients $a_{a}^{i, h, n}, b_{a}^{i, h, n}, c_{a}^{i, h, n}$ can be obtained by determining the partial derivatives of the BPR link performance function:

$$
\begin{gathered}
a_{a}^{i, h, n}=\left.\frac{\partial t_{a}^{i}}{\partial v_{a}^{i}}\right|_{\left(K_{a, h}^{i}, L_{a, n}\right)} \\
b_{a}^{i, h, n}=\left.\frac{\partial t_{a}^{i}}{\partial x_{a}}\right|_{\left(K_{a, h}^{i}, L_{a, n}\right)} \\
c_{a}^{i, h, n}=t_{a}^{i}\left(K_{a, h}^{i}, L_{a, n}\right)-K_{a, h}^{i} \cdot a_{a}^{i, h, n}-L_{a, n} \cdot b_{a}^{i, h, n} .
\end{gathered}
$$

It is clear that link travel time can be precisely estimated by (13), if very large $H, N$ are set to guarantee sufficient number of binary space splits. The link travel time function thus can be replaced by an equivalent set of mixed integer linear constraints. For link $a, a \in A(h=1, \ldots, H ; n=$ $1, \ldots, N ; i=m, e)$, we have

$$
\begin{gathered}
L \cdot \xi_{a, h}^{i} \leq v_{a}^{i}-K_{a, h}^{i} \leq U \cdot\left(1-\xi_{a, h}^{i}\right)-\varepsilon \\
\kappa_{a, h}^{i}=\xi_{a, h+1}^{i}-\xi_{a, h}^{i} \\
L \cdot \tau_{a, n} \leq x_{a}-L_{a, n} \leq U \cdot\left(1-\tau_{a, n}\right)-\varepsilon \\
\lambda_{a, n}=\tau_{a, n+1}-\tau_{a, n} \\
\psi_{a}^{i, h, n}=\kappa_{a, h}^{i}+\lambda_{a, n} \\
L \cdot\left(2-\psi_{a}^{i, h, n}\right) \leq t_{a}-\left(a_{a}^{i, h, n} \cdot v_{a}^{i}+b_{a}^{i, h, n} \cdot x_{a}+c_{a}^{i, h, n}\right) \\
\leq U \cdot\left(2-\psi_{a}^{i, h, n}\right) \\
\text { integer } \xi_{a, h}^{i}, \tau_{a, n} \in\{0,1\},
\end{gathered}
$$

where $L, U$ are, respectively, a very large negative constant and a very large positive constant; $\varepsilon$ is a very small positive constant. It is not difficult to prove that (15) is equivalent to the linear approximation function (13). We here do not attempt to present the proof again because the detailed proof can be found in [27].

3.2. Linearization of the Constraints. The proposed NDP model includes three kinds of constraints: deterministic user equilibrium constraint, definitional constaints (e.g., demand conservation) and other side constraints (invested budget constaint and boundary constraints of design variables).

(1) Deterministic User Equilibrium Constraint. Recall that the deterministic user equilibrium principle can also be expressed by complementary constraint, which is derived from the first-order condition of the lower level traffic assignment problem:

$$
f_{r, w}^{i} \cdot\left(c_{r, w}^{i}-\mu_{w}^{i}\right)=0, \quad c_{r, w}^{i}-\mu_{w}^{i}=0, \quad \forall r, w, i .
$$

The "if-then" complementary constraint can be transformed into an equivalent set of constraints by introducing a set of binary variables, shown as:

$$
\begin{gathered}
L \cdot \sigma_{r, w}^{i}+\varepsilon \leq f_{r, w}^{i} \leq U \cdot\left(1-\sigma_{r, w}^{i}\right) \\
L \cdot \sigma_{r, w}^{i} \leq c_{r, w}^{i}-\mu_{w}^{i} \leq U \sigma_{r, w}^{i} \\
c_{r, w}^{i}-\mu_{w}^{i} \geq 0
\end{gathered}
$$

integer $\sigma_{r, w}^{i} \in\{0,1\}, \quad i=\{m, e\}$.

Evidently, in (17), if $\sigma_{r, w}^{i}=0$, we have $f_{r, w}^{i}>0$ and $c_{r, w}^{i}-$ $\mu_{w}^{i}=0$; otherwise, $\sigma_{r, w}^{i}=1$, we have $f_{r, w}^{i}=0$ and $c_{r, w}^{i}-\mu_{w}^{i} \geq 0$. That is, the user equilibrium condition holds.

(2) Definitional Constaints. We have

$$
\begin{gathered}
\sum_{r \in R_{w}^{i}} f_{r, w}^{i}=q_{w}^{i} \\
v_{a}^{i}=\sum_{w \in W^{i}} \sum_{r \in R_{w}^{i}} f_{r, w}^{i} \delta_{a r, w}^{i} \\
c_{r, w}^{i}=\sum_{a \in A} t_{a}^{i}\left(v_{a}^{i}, x_{a}\right) \delta_{a r, w}^{i} \\
v_{a}^{i} \geq 0, \quad f_{r, w}^{i} \geq 0, \\
t_{a}^{i} \geq t_{a}^{0}, \quad i \in\{m, e\} .
\end{gathered}
$$

The definitional constraints in (18) are all linear constraints due to their additive properties.

(3) Other Side Constraints. We have

$$
0 \leq x_{a} \leq x_{a}^{u}, \quad 0 \leq \sum_{a \in \bar{A}} \gamma_{a} x_{a} \leq B .
$$

So far, we have completely transformed the lower level user equilibrium traffic assignment problem into an equivalent set of mixed integer linear constraints. Since the design objective function (1) is also linear for the NDP with fixed demand, the bilevel NDP model simultaneously considering morning and evening peak-hour demands can be perfectly transformed into the equivalent MILP.

3.3. Solution Algorithm. Comparing to a nonlinear and nonconvex bilevel NDP, it is simple and effective to solve the transformed MILP problem. A more attractive merit of solving the NDP problem by transforming into MILP is that a global solution can be guaranteed. The global optimal solution is helpful and convincing for exploring 
TABLE 1: O-D demands in two peaks.

\begin{tabular}{lcccc}
\hline O-D pair & $1 \rightarrow 3$ & $3 \rightarrow 1$ & $2 \rightarrow 3$ & $3 \rightarrow 2$ \\
\hline $\begin{array}{l}\text { Commute demand } \\
\text { in the morning peak }\left(q_{w}^{m}\right)\end{array}$ & 25 & 10 & 20 & 10 \\
$\begin{array}{l}\text { Commute demand } \\
\text { in the evening peak }\left(q_{w}^{e}\right)\end{array}$ & 10 & 25 & 10 & 20 \\
\hline
\end{tabular}

TABLE 2: Parameters used for numerical examples.

\begin{tabular}{lcccc}
\hline Link number & 1 & 2 & 3 & 4 \\
\hline$t_{a}^{0}$ & 5.0 & 10.0 & 15.0 & 10.0 \\
$c_{a}^{0}$ & 15 & 20 & 20 & 25 \\
$\gamma_{a}$ & 5.0 & 10.0 & 15.0 & 10.0 \\
\hline
\end{tabular}

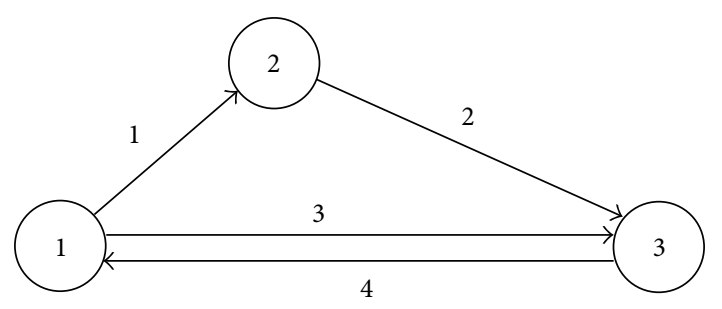

FIGURE 3: The transportation network used in numerical examples.

the design effect of the proposed NDP model. The MILP model can easily be solved by off-the-shelf MIP solvers, including IBM ILOG CPLEX, LINGO, and GUROBI. It has been demonstrated that the bilevel optimization problem can be fully transformed into equivalent MILP and solved by the MIP solvers efficiently and precisely (e.g., [27]) [29]. In this paper, the proposed bilevel NDP model will be solved as the equivalent MILP by the CPLEX solver.

\section{Numerical Examples}

4.1. Preliminary. The numerical examples are used to illustrate the difference between the proposed NDP model and the traditional one considering traffic patterns in one peak. In the numerical study, a small network shown in Figure 3 is used to demonstrate the property of the proposed model. This transportation network comprises of 3 nodes, 4 links, and 4 O-D pairs in each commute peak. All 4 links are considered in the candidate set of the capacity improvement scheme, namely, $\bar{A}=A$. The travel demands for each O-D pair in morning and evening peaks are given in Table 1. Table 2 provides the link performance parameters, $t_{a}^{0}$ and $c_{a}^{0}$, and the link capacity expansion cost coefficient $\gamma_{a}$. The weighted parameters in design objective function are set as $\alpha=0.5$, $\beta=0.5$. The total budget invested on the link capacity improvement scheme is 300 . The commercial optimization package CPLEX-12.5 is used to solve the MILP model with a gap tolerance of $0.1 \%$. All experiments run on Windows 7 system with the following attributes: Intel Core i5-2520 $2.5 \mathrm{GHz} \times 2$ and $4 \mathrm{~GB}$ RAM.
4.2. Comparison of the NDP Schemes. We investigate three NDP schemes and make a comparison of them in terms of network design effect. These NDP schemes are the proposed NDP simultaneously considering morning and evening peakhour commuting demands, the traditional NDP only considering morning peak-hour demand matrix, and the traditional NDP only considering evening peak-hour demand matrix. The outcomes of three NDP schemes are provided in Tables 3,4 , and 5 , respectively.

In the NDP considering morning peak-hour demand, the total travel time in the morning peak is 860.551 . These four links will be improved by adding capacities of 9.749, $18.202,2.998$, and 2.427 , respectively. Once this NDP scheme is implemented, the total travel time for the network users commuting in the evening peak is 1296.560 . It can be found that travelers of O-D pairs 1-3 and 2-3 will largely benefit from Scheme A in the morning and evening peaks. But the travelers of another two O-D pairs obtain little benefit from the NDP Scheme A.

In the NDP considering evening peak-hour demand, the total travel time in the evening peak is 851.822 . Links 1 and 4 will be expanded by adding capacities of 7.637 and 26.181, respectively, and links 2 and 3 maintain their initial capacities. Once the NDP Scheme B is performed, the total travel time for the network users commuting in the morning peak is 958.753. We can see that travelers of O-D pairs 1-3, 3-1, and 3-2 will benefit from Scheme A in two commute peaks in that their path travel times will be reduced by the NDP scheme. But Scheme B does not bring any benefit for the travelers of O-D pair 2-3.

In the NDP simultaneously considering two peak-hour demands, the total travel time in the morning peak is 902.092 and the total travel time in the evening peak is 877.038. Links 1,2, and 4 will be expanded by adding capacities of $6.152,6.201$, and 20.725, respectively, and link 3 will not be considered to be improved. It is easy to see that travelers of all O-D pairs will benefit from the NDP Scheme C in two commute peaks.

By comparing the NDPs considering one peak-hour demand, namely, Schemes A and B, it is shown that Scheme $B$ is better than Scheme A in terms of reducing total travel time on the network. Specifically, the sum of total travel time of two peaks in Scheme B is far less than that in Scheme $A$ in the sense that the travelers commuting in the evening peak largely reduce their travel costs in Scheme B. This clearly indicates that the effects of the NDPs considering one peak-hour demand will be affected by the network structure and link sensitivity, which bring some troubles in choosing appropriate peak-hour demand matrix. The fact that Scheme $\mathrm{A}$ is far inferior to Scheme $\mathrm{B}$ on design effect also gives us a reminder that it should be careful to select the commute peak in performing traffic data collection.

We also compare the performance of the NDP simultaneously considering two peak-hour demands (Scheme C) with the NDP considering one peak-hour demand (Scheme B). As expected, the sum of total travel time of two peaks in Scheme C is less than that in Scheme B, although total travel time in the evening peak of Scheme $\mathrm{C}$ is slightly more than that of Scheme B. The reason is that Scheme B overlooks 
TABle 3: Network design considering morning peak-hour demand (Scheme A).

\begin{tabular}{|c|c|c|c|c|c|c|c|c|}
\hline \multirow{2}{*}{ Commute peak } & \multirow{2}{*}{ TTC } & \multicolumn{3}{|c|}{ Link } & \multicolumn{4}{|c|}{ Path } \\
\hline & & Number & Expanded capacity $\left(x_{a}\right)$ & Flow $\left(v_{a}^{i}\right)$ & Number & Link component & Flow $\left(f_{r, w}^{i}\right)$ & Equilibrium cost $\left(\mu_{w}^{i}\right)$ \\
\hline \multirow{5}{*}{ Morning peak } & \multirow{5}{*}{860.551} & 1 & 9.749 & 18.108 & 1 & $1-2$ & 8.108 & 15.655 \\
\hline & & 2 & 18.202 & 28.108 & 2 & 3 & 16.892 & 15.655 \\
\hline & & 3 & 2.998 & 16.892 & 3 & 4 & 10.000 & 10.424 \\
\hline & & 4 & 2.427 & 20.000 & 4 & 2 & 20.000 & 10.440 \\
\hline & & - & - & - & 5 & $1-4$ & 10.000 & 15.639 \\
\hline \multirow{5}{*}{ Evening peak } & \multirow{5}{*}{1296.560} & 1 & 9.749 & 20.000 & 1 & $1-2$ & 0.000 & 15.327 \\
\hline & & 2 & 18.202 & 10.000 & 2 & 3 & 10.000 & 15.080 \\
\hline & & 3 & 2.998 & 10.000 & 3 & 4 & 25.000 & 20.873 \\
\hline & & 4 & 2.427 & 45.000 & 4 & 2 & 10.000 & 10.007 \\
\hline & & - & - & - & 5 & $1-4$ & 20.000 & 26.193 \\
\hline Sum & 2157.111 & - & - & - & - & - & - & - \\
\hline
\end{tabular}

TABLE 4: Network design considering evening peak-hour demand (Scheme B).

\begin{tabular}{|c|c|c|c|c|c|c|c|c|}
\hline \multirow{2}{*}{ Commute peak } & \multirow{2}{*}{ TTC } & \multicolumn{3}{|c|}{ Link } & \multicolumn{4}{|c|}{ Path } \\
\hline & & Number & Expanded capacity $\left(x_{a}\right)$ & Flow $\left(v_{a}^{i}\right)$ & Number & Link component & Flow $\left(f_{r, w}^{i}\right)$ & Equilibrium cost $\left(\mu_{w}^{i}\right)$ \\
\hline \multirow{5}{*}{ Morning peak } & \multirow{5}{*}{958.753} & 1 & 7.637 & 13.551 & 1 & $1-2$ & 3.551 & 17.980 \\
\hline & & 2 & 0.000 & 23.551 & 2 & 3 & 21.449 & 17.979 \\
\hline & & 3 & 0.000 & 21.449 & 3 & 4 & 10.000 & 10.035 \\
\hline & & 4 & 26.181 & 20.000 & 4 & 2 & 20.000 & 12.884 \\
\hline & & - & - & - & 5 & $1-4$ & 10.000 & 15.131 \\
\hline \multirow{5}{*}{ Evening peak } & \multirow{5}{*}{851.822} & 1 & 7.637 & 20.000 & 1 & $1-2$ & 0.000 & 15.551 \\
\hline & & 2 & 0.000 & 10.000 & 2 & 3 & 10.000 & 15.141 \\
\hline & & 3 & 0.000 & 10.000 & 3 & 4 & 25.000 & 10.896 \\
\hline & & 4 & 26.181 & 45.000 & 4 & 2 & 10.000 & 10.094 \\
\hline & & - & - & - & 5 & $1-4$ & 20.000 & 16.353 \\
\hline Sum & 1810.575 & - & - & - & - & - & - & - \\
\hline
\end{tabular}

the traffic congestion in the morning peak; that is, it does not take into account the benefits of the travelers in morning peak while designing NDP scheme. In reality, Schemes A and $\mathrm{B}$ can be regarded as two special cases of the proposed model if one of the weighted parameters is zero. In summary, the proposed NDP model simultaneously considering two peak-hour demands can well characterize the practical traffic situation and also bring about promising design effect in terms of improving the transportation system performance. Note that the NDP model can be extended to consider more than two peaks. But the NDP simultaneously considering two peak-hour demands is believed to be good enough when the data collection costs in each peak and model flexibility are taken into account.

4.3. Impact Analysis of Weighted Parameters. We here conduct the impact analysis of the weighted parameter setting for the design objective function. Without loss of generality, it is assumed that $\alpha+\beta=1.0$ by normalization. The weighted parameter $\beta$ is set to be increased from 0.0 to 1.0 with each increment of 0.1 . The variation of total travel time for each peak with different weighted parameter setting is depicted in
Figure 4. As shown in Figure 5, the total travel time of two peaks changes with the weighted parameter setting.

It is shown in Figure 4 that, as expected, the total travel time of evening peak decreases monotonically with the increasing weight of $\beta$, since an increasing priority will be put on improving the traffic congestion in the evening peak. In turn, the total travel time of morning peak continuously increases with the weighted parameter $\beta$; that is, less emphasis will be paid on reducing the traffic congestion in the morning peak. It should be stressed that, the improvement effect of the NDP scheme in great measure depends on the network structure and demand distribution. In this regard, we repeat that the NDP scheme A greatly overlooks the social welfare of the travelers in the evening peak. Therefore, the network planner should avoid implementing the NDP scheme A.

In Figure 5, we can clearly see how important it is to account for the traffic congestions in both commuting peaks. Although the weight of considering the traffic pattern in the evening peak is small (e.g., $\beta=0.1$ ), the network performance for whole daily commuting will be greatly improved. That is, the NDP only considering morning peak-hour 
TABLE 5: Network design simultaneously considering two peak-hour demands (Scheme C).

\begin{tabular}{|c|c|c|c|c|c|c|c|c|}
\hline \multirow{2}{*}{ Commute peak } & \multirow{2}{*}{ TTC } & \multicolumn{3}{|c|}{ Link } & \multicolumn{4}{|c|}{ Path } \\
\hline & & Number & Expanded capacity $\left(x_{a}\right)$ & Flow $\left(v_{a}^{i}\right)$ & Number & Link component & Flow $\left(f_{r, w}^{i}\right)$ & Equilibrium cost $\left(\mu_{w}^{i}\right)$ \\
\hline \multirow{5}{*}{ Morning peak } & \multirow{5}{*}{902.092} & 1 & 6.152 & 16.198 & 1 & $1-2$ & 6.198 & 16.757 \\
\hline & & 2 & 6.201 & 26.198 & 2 & 3 & 18.804 & 16.758 \\
\hline & & 3 & 0.000 & 18.804 & 3 & 4 & 10.000 & 10.055 \\
\hline & & 4 & 20.725 & 20.000 & 4 & 2 & 20.000 & 11.499 \\
\hline & & - & - & - & 5 & $1-4$ & 10.000 & 15.313 \\
\hline \multirow{5}{*}{ Evening peak } & \multirow{5}{*}{877.038} & 1 & 6.152 & 20.000 & 1 & $1-2$ & 0.000 & 15.631 \\
\hline & & 2 & 6.201 & 10.000 & 2 & 3 & 10.000 & 15.141 \\
\hline & & 3 & 0.000 & 10.000 & 3 & 4 & 25.000 & 11.407 \\
\hline & & 4 & 20.725 & 45.000 & 4 & 2 & 10.000 & 10.032 \\
\hline & & - & - & - & 5 & $1-4$ & 20.000 & 17.007 \\
\hline Sum & 1779.130 & - & - & - & - & - & - & - \\
\hline
\end{tabular}

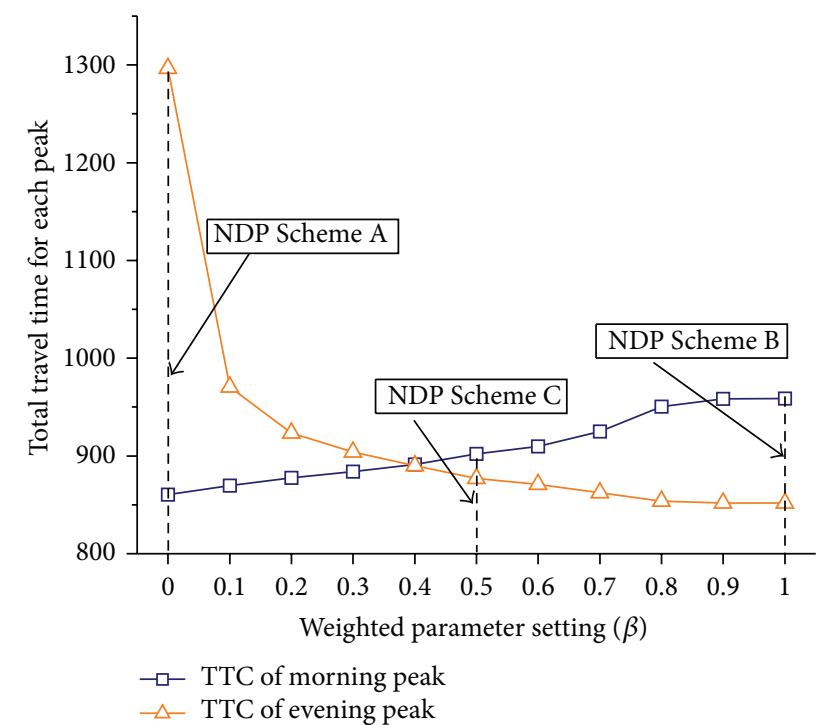

FIGURE 4: Total travel time for each peak with different weighted parameter settings.

demand is far inferior to the NDPs taking into account the traffic congestion in the evening peak. Meanwhile, it can be found that how to determine the weighted parameter setting is important for developing a reasonable NDP scheme. Recall that the network decision-maker only concerns the traffic congestions in two commuting peaks, although some travelers might be more sensitive to the traffic congestion in the morning. To achieve the design objective of improving the traffic situation of two peaks as much as possible, the weighted parameter setting is preferred to set as $\alpha=\beta$, $\alpha, \beta>0$, no matter whether the weighted parameters are normalized or not. In other words, if $\alpha=\beta, \alpha, \beta>0$, the NDP considering two peak-hour demands always performs no worse than other NDP schemes (including the NDPs considering one peak-hour demand) in terms of minimizing the total travel time of two commuting peaks. It is not difficult to verify this conclusion. By revisiting the design objective function in (1), it can be seen that, when $\alpha=\beta, \alpha, \beta>0$,

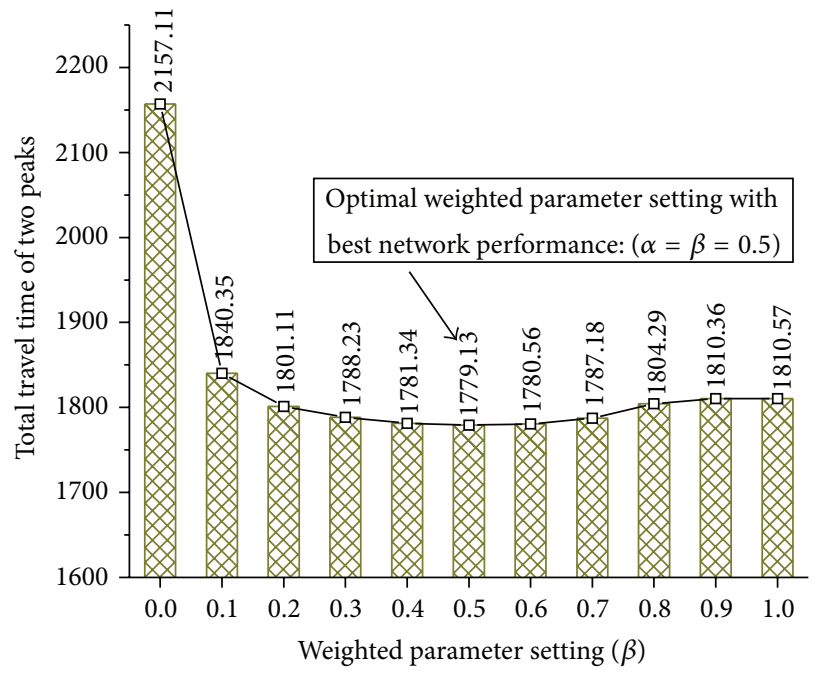

FIGURE 5: Total travel time of two peaks with different weighted parameter settings.

the design objective function is to directly optimize the network performance in terms of total travel time of two peaks, with no error due to introducing priority of any peak. The outcome in the numerical examples also indicates that NDP scheme will have the best effect when $\alpha=\beta=0.5$.

\section{Conclusions and Further Studies}

In the previous NDP studies, it was always assumed that the NDP considering one peak-hour demand distribution is capable and effective to characterize real traffic situation on the network. However, we found that the NDP model considering only one peak-hour demand matrix might be unable to describe the real traffic patterns due to the asymmetric traffic characteristics in the morning and evening peaks, and the impacts of network structure and link sensitivity. The traditional NDP model considering one peakhour demand matrix thus may lead to misleading outcomes for transportation management and planning. To address 
these problems, we proposed an NDP model simultaneously considering morning and evening peak-hour demands. The proposed model can be used to some extent to avoid the impacts of asymmetric demand distributions in the morning and evening commute peaks, the network structure, and link sensitivity. The NDP problem is formulated as a bilevel programming model, in which the upper level is to minimize the weighted sum of total travel time for the network users travelling in both morning and evening commute peaks, and the lower level is to characterize the user equilibrium choice behaviors of the travelers in two peaks. Some extensions on elastic and uncertain peak-hour demands for two peaks are also discussed. The proposed NDP model is transformed into an equivalent MILP, which can be solved by optimization solvers (e.g., CPLEX). Through this transformation, a global solution can be guaranteed. Numerical examples are finally performed to demonstrate the effectiveness of the developed model. It was shown that the proposed NDP model can generate more promising design effect than the traditional NDP model considering one peak-hour demand and avoid the misleading decision. Meanwhile, it was found that how to choose a surveyed peak for data collection is very important for the traditional NDP model considering one peak-hour demand. That is, we should carefully determine which peakhour demand is less likely to result in misleading outcomes. Furthermore, for weighted parameter setting, we recommend to set $\alpha=\beta, \alpha, \beta>0$ which leads to the best network design effect.

Further studies could be carried out to extend the proposed model in the following aspects. First, although we introduce a conceptual framework of the NDP considering peak-hour uncertain demands in two peaks, a tractable and specific NDP model considering two peak-hour uncertain demands could be an interesting work. Second, the distribution of multiclass users with different value-of-times and mixed vehicles would also show a large diversity in the morning and evening commute peaks. To address such diversity in the NDP problem reveals important investigations.

\section{Notations}

\section{Design Variables}

$\mathbf{x v}=\left(\ldots, x_{a}, \ldots\right), a \in \bar{A}$ : Design variables, namely, link capacity expansions.

Variables to Be Determined in Each Equilibrium

$v_{a}^{m}, v_{a}^{e}: \quad$ Flow on link $a \in A$ in the morning peak and evening peak, respectively

$t_{a}^{m}\left(v_{a}^{m}\right), t_{a}^{e}\left(v_{a}^{e}\right):$ Travel time on link $a \in A$ in the morning peak and evening peak, respectively

$f_{r, w}^{m}: \quad$ Traffic flow on route $r \in R_{w}^{m}, w \in W^{m}$ in the morning peak

$f_{r, w}^{e}: \quad$ Traffic flow on route $r \in R_{w}^{e}, w \in W^{e}$ in the evening peak $c_{r, w}^{i}$ : Travel time on route $r$ for O-D pair $w$ in the morning if $i=m$ or in the evening peak if $i=e$

$\mu_{w}^{m}: \quad$ Equilibrium minimal travel cost between O-D pair $w \in W^{m}$ in the morning peak

$\mu_{w}^{e}: \quad$ Equilibrium minimal travel cost between O-D pair $w \in W^{e}$ in the evening peak

$b_{a}\left(x_{a}\right)$ : Construction cost for each improved link $a \in \bar{A}$.

\section{Parameter Given}

a: $\quad$ Link $a \in A$

$\bar{A}: \quad$ Set of candidate links to be improved, $\bar{A} \subset A$

$W^{m}, W^{e}$ : Sets of O-D pairs in morning and even peaks respectively

$w: \quad$ O-D pair $w \in W^{m} \cup W^{e}$

$R_{w}^{m}$ : Set of routes connecting O-D pair $w \in W^{m}$ in morning commute peak

$R_{w}^{e}$ : Set of routes connecting O-D pair $w \in W^{e}$ in evening commute peak

$r: \quad$ Route $r \in R_{w}^{m} \cup R_{w}^{e}$

$\delta_{a r, w}^{m}$ : Link-route indicator $\delta_{a r, w}^{m}$ equals 1 if route $r$ between O-D pair $w \in W^{m}$ uses link $a \in A$ at morning peak, and 0 otherwise

$\delta_{a r, w}^{e}: \quad$ Link-route indicator $\delta_{a r, w}^{e}$ equals 1 if route $r$ between O-D pair $w \in W^{e}$ uses link $a \in A$ at evening peak, and 0 otherwise

$q_{w}^{m}, q_{w}^{e}: \quad$ Travel demand for O-D pair in the morning peak and evening peak respectively

$\alpha, \beta: \quad$ Weighted parameter in objective function

$t_{a}^{0}: \quad$ Free flow travel time for link $a \in A$

$c_{a}^{0}$ : $\quad$ Existing capacity for link $a \in A$

$\gamma_{a}$ : Parameter in link capacity improvement construction cost.

\section{Conflict of Interests}

All the authors of the paper declare that there is no conflict of interests regarding the publication of this paper.

\section{Acknowledgments}

This study is supported by a program for new century excellent talents in university (Grant no. NCET-10-0637), a research Grant for 2013 Shanghai Postdoctoral Sustentation Fund (no. 13R21416400), and a research Grant for China Postdoctoral Science Foundation (no. 2013M531222).

\section{References}

[1] Q. Meng, H. Yang, and M. G. H. Bell, "An equivalent continuously differentiable model and a locally convergent algorithm for the continuous network design problem," Transportation Research Part B, vol. 35, no. 1, pp. 83-105, 2001.

[2] M. Abdulaal and L. J. LeBlanc, "Continuous equilibrium network design models," Transportation Research Part B, vol. 13, no. 1, pp. 19-32, 1979.

[3] T. L. Magnanti and R. T. Wong, "Network design and transportation-planning-models and algorithms," Transportation Science, vol. 18, no. 1, pp. 1-55, 1984. 
[4] H. Yang and M. G. H. Bell, "Models and algorithms for road network design: a review and some new developments," Transport Reviews, vol. 18, no. 3, pp. 257-278, 1998.

[5] A. Karoonsoontawong and S. Travis Waller, "Comparison of system- and user-optimal stochastic dynamic network design models using Monte Carlo bounding techniques," Transportation Research Record, vol. 1923, pp. 91-102, 2005.

[6] S. V. Ukkusuri and S. T. Waller, "Linear programming models for the user and system optimal dynamic network design problem: formulations, comparisons and extensions," Networks and Spatial Economics, vol. 8, no. 4, pp. 383-406, 2008.

[7] A. Chen and X. Xu, "Goal programming approach to solving network design problem with multiple objectives and demand uncertainty," Expert Systems with Applications, vol. 39, no. 4, pp. 4160-4170, 2012.

[8] A. Chen and C. Yang, "Stochastic transportation network design problem with spatial equity constraint," Transportation Research Record, vol. 1882, pp. 97-104, 2004.

[9] H. K. Lo, X. W. Luo, and B. W. Y. Siu, "Degradable transport network: travel time budget of travelers with heterogeneous risk aversion," Transportation Research Part B, vol. 40, no. 9, pp. 792806, 2006.

[10] H. Shao, W. H. K. Lam, Q. Meng, and M. L. Tam, "Demanddriven traffic assignment problem based on travel time reliability," Transportation Research Record, vol. 1985, pp. 220-230, 2006.

[11] H. Wang, W. H. K. Lam, X. Zhang, and H. Shao, "Sustainable transportation network design with stochastic demands and chance constraints," International Journal of Sustainable Transportation, 2013.

[12] H. K. Lo and W. Y. Szeto, "Time-dependent transport network design: a study of budget sensitivity," Journal of the Eastern Asia Society For Transportation Studies, vol. 5, pp. 1124-1139, 2003.

[13] H. K. Lo and W. Y. Szeto, “Time-dependent transport network design under cost-recovery," Transportation Research Part B, vol. 43, no. 1, pp. 142-158, 2009.

[14] W. Y. Szeto and H. K. Lo, “Time-dependent transport network improvement and tolling strategies," Transportation Research Part A, vol. 42, no. 2, pp. 376-391, 2008.

[15] S. V. Ukkusuri and G. Patil, "Multi-period transportation network design under demand uncertainty," Transportation Research Part B, vol. 43, no. 6, pp. 625-642, 2009.

[16] S. T. Waller, K. C. Mouskos, D. Kamaryiannis, and A. K. Ziliaskopoulos, "A linear model for the continuous network design problem," Computer-Aided Civil and Infrastructure Engineering, vol. 21, no. 5, pp. 334-345, 2006.

[17] S. T. Waller and A. K. Ziliaskopoulos, "Stochastic dynamic network design problem," Transportation Research Record, vol. 1771, pp. 106-113, 2001.

[18] Q. Meng, H. Yang, and H. Wang, "The transportation network design problems," Transportation Research Part B, 2013.

[19] A. Chen, Z. Zhou, P. Chootinan, S. Ryu, C. Yang, and S. C. Wong, "Transport network design problem under uncertainty: a review and new developments," Transport Reviews, vol. 31, no. 6, pp. 743-768, 2011.

[20] Highway Capacity Manual, "Transportation Research Board," Washington, DC, USA, 2000.

[21] X. Zhang, H. Yang, H.-J. Huang, and H. M. Zhang, "Integrated scheduling of daily work activities and morning-evening commutes with bottleneck congestion," Transportation Research Part A, vol. 39, no. 1, pp. 41-60, 2005.
[22] X. Zhang, H.-J. Huang, and H. M. Zhang, "Integrated daily commuting patterns and optimal road tolls and parking fees in a linear city," Transportation Research Part B, vol. 42, no. 1, pp. 38-56, 2008.

[23] X. Zhang and B. van Wee, "Efficiency comparison of various parking charge schemes considering daily travel cost in a linear city," European Journal of Transport and Infrastructure Research, vol. 11, no. 2, pp. 234-255, 2011.

[24] R. Venkatanarayana, B. L. Smith, and M. J. Demetsky, "Quantum-frequency algorithm for automated identification of traffic patterns," Transportation Research Record, vol. 2024, pp. 8-17, 2007.

[25] X. Zhang, "Effects of queue spillover in networks considering simultaneous departure time and route choices," Transportation Planning and Technology, vol. 36, no. 3, pp. 267-286, 2013.

[26] Y. Sheffi, Urban Transportation Networks: Equilibrium Analysis with Mathematical Programming Methods, Prentice Hall, Englewood Cliff, NJ, USA, 1985.

[27] D. Z. W. Wang and H. K. Lo, "Global optimum of the linearized network design problem with equilibrium flows," Transportation Research Part B, vol. 44, no. 4, pp. 482-492, 2010.

[28] P. Luathep, A. Sumalee, W. H. K. Lam, Z.-C. Li, and H. K. Lo, "Global optimization method for mixed transportation network design problem: a mixed-integer linear programming approach," Transportation Research Part B, vol. 45, no. 5, pp. 808-827, 2011.

[29] X. Zhang and B. van Wee, "Enhancing transportation network capacity by congestion pricing with simultaneous toll location and toll level optimization," Engineering Optimization, vol. 44, no. 4, pp. 477-488, 2012. 


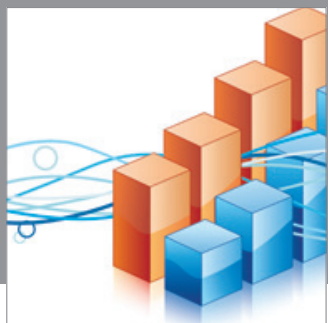

Advances in

Operations Research

mansans

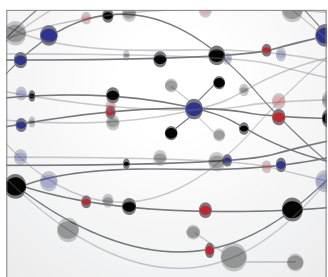

The Scientific World Journal
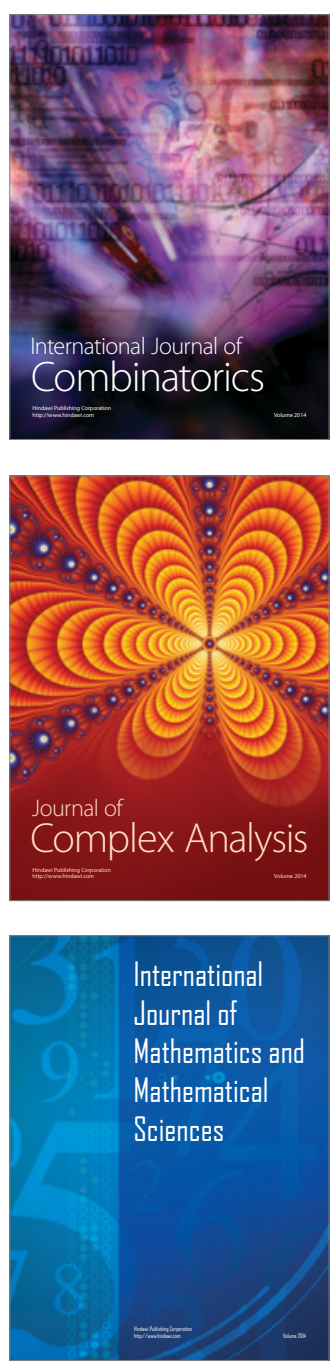
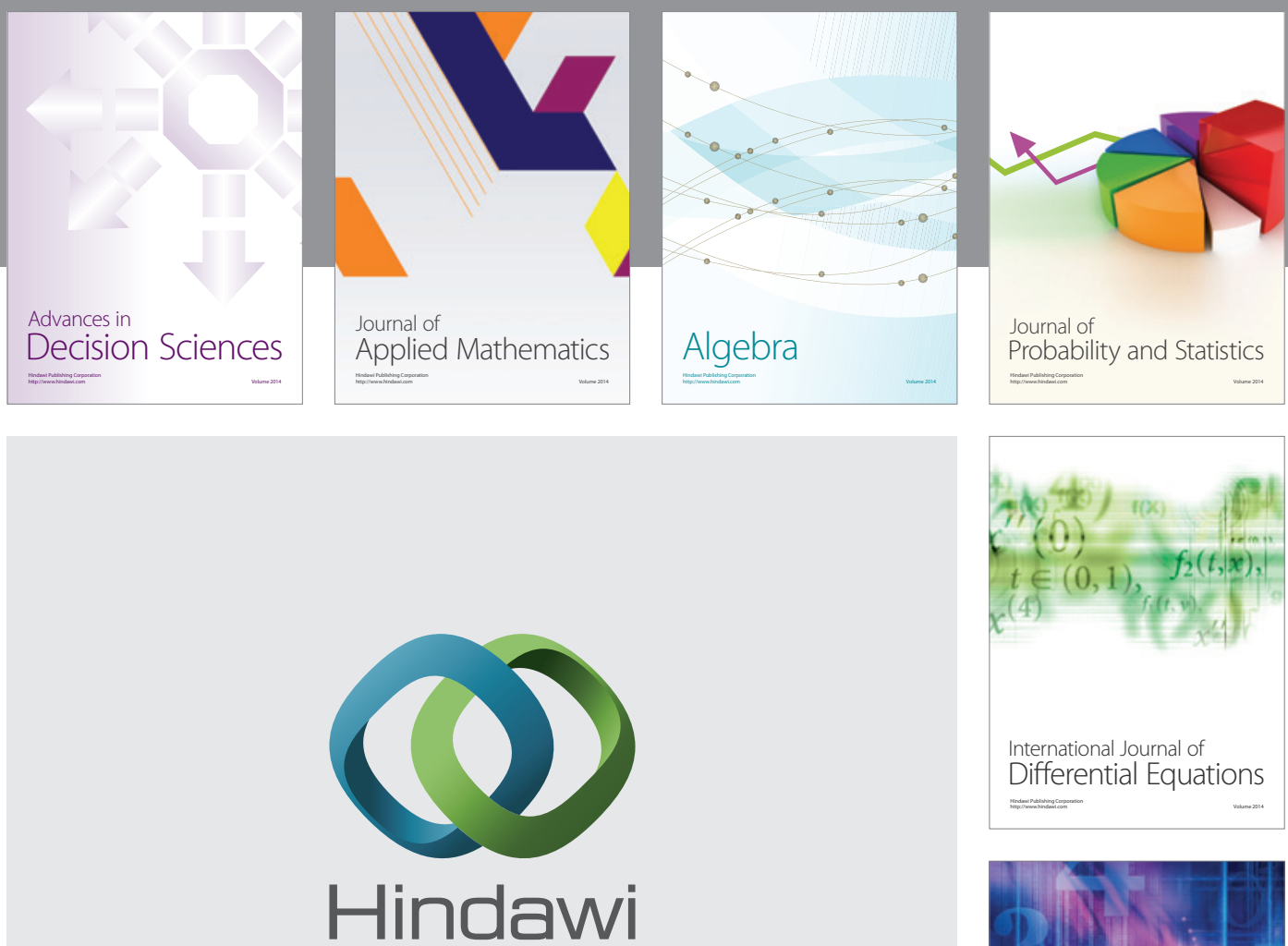

Submit your manuscripts at http://www.hindawi.com
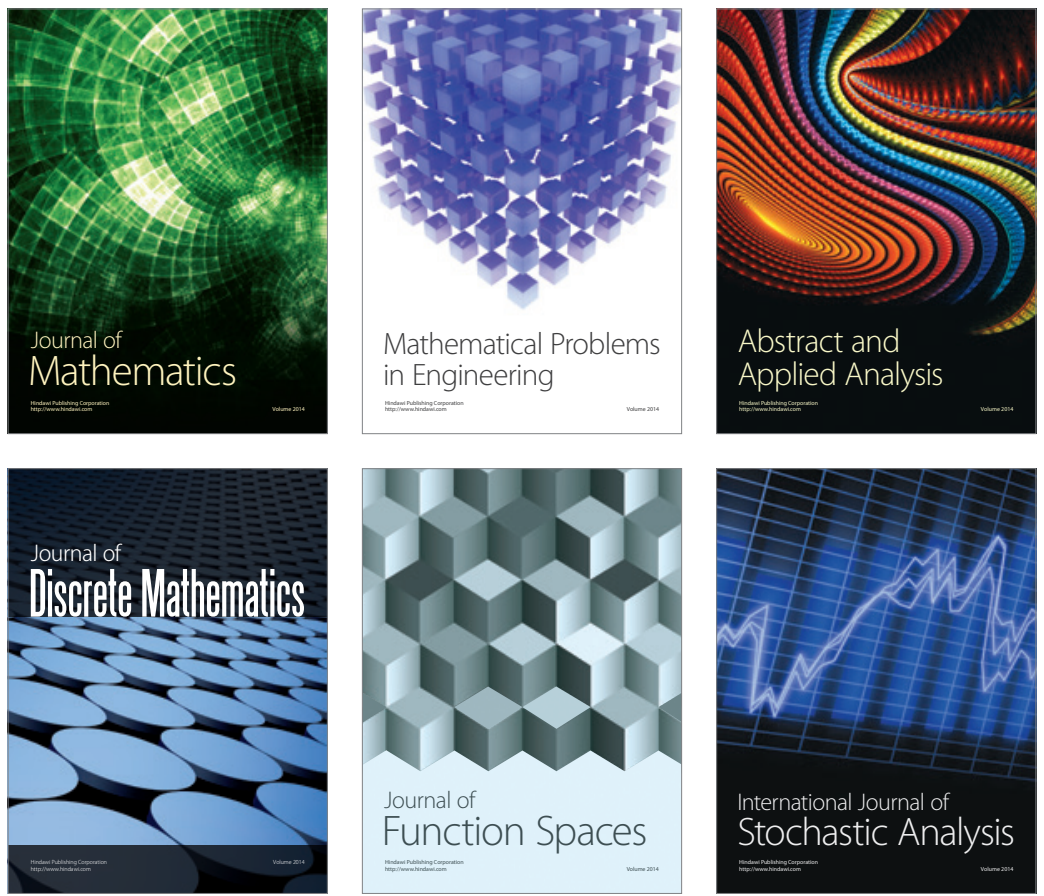

Journal of

Function Spaces

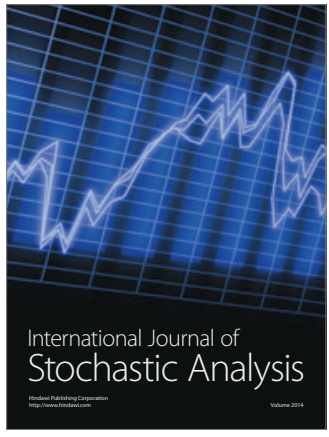

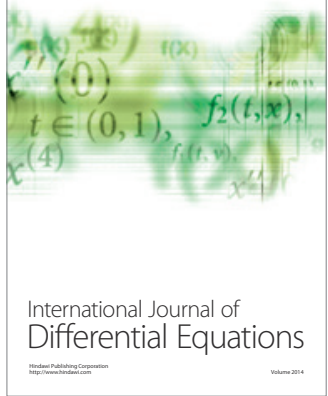
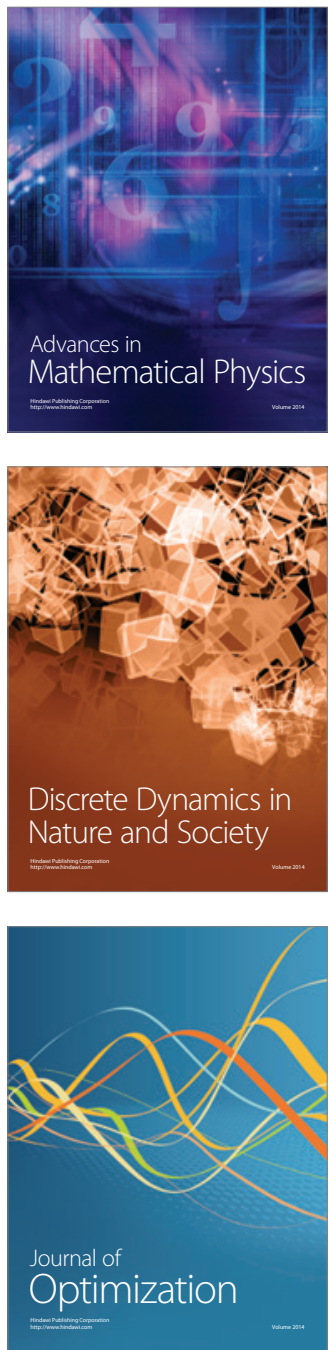